\title{
Role of Ultrasonographic Measurement of Fetal Kidney Length in Determination of Gestational Age during Third Trimester of Pregnancy
}

\author{
Mahmoud Mohamed Ghaleb*, Afnan Ibrahim Abdel Fatah Shokri, \\ Mohammed Salah El Sayed El Sokkary, Maged Mahmoud Ali El Shourbagy \\ Department of Obstetrics and Gynecology, Faculty of Medicine, Ain Shams University, Cairo, Egypt \\ Email: *mahmoudghaleb203050@gmail.com
}

How to cite this paper: Ghaleb, M.M., Shokri, A.I.A.F., El Sayed El Sokkary, M.S. and El Shourbagy, M.M.A. (2021) Role of Ultrasonographic Measurement of Fetal Kidney Length in Determination of Gestational Age during Third Trimester of Pregnancy. Open Journal of Obstetrics and Gynecology, 11, 221-232.

https://doi.org/10.4236/ojog.2021.113022

Received: February 7, 2021

Accepted: March 2, 2021

Published: March 5, 2021

Copyright $\odot 2021$ by author(s) and Scientific Research Publishing Inc. This work is licensed under the Creative Commons Attribution International License (CC BY 4.0).

http://creativecommons.org/licenses/by/4.0/

\begin{abstract}
Background: In routine ultrasonography, the ultrasonologist measures the bi-parietal diameter (BPD), head circumference (HC), abdominal circumference (AC) and femur length (FL) in estimating the gestational age (GA) and estimated date of delivery. However, as the pregnancy advances these parameters become increasingly unreliable in prediction of GA. Estimation of GA in late second and third trimester accurately still a problem till now. Fetal kidney has been shown a steady growth of $1.7 \mathrm{~mm}$ fortnightly (every 2 weeks) along pregnancy and is unaffected by growth abnormalities. Many studies have reported that fetal kidney length (FKL) correlates with the gestational age in late trimester very strong. The study aimed to assess the accuracy of the gestational age estimated by mean fetal kidney length compared to multiple growth parameters like BPD, HC, AC \& FL in addition to the actual gestational age derived from the reliable last menstrual period. Methods: Cross sectional hospital based study, was conducted at ultrasound unit; department of Obstetrics and Gynecology, Faculty of Medicine, Ain Shams University from the period of July 2018 to December 2018. A total number of 115 Pregnant women at their third trimester by reliable LMP attended antenatal care clinic at Ain-Shams University Hospital and consented to participate in this study. Every woman was subjected to: Expected date of delivery was calculated according to Naegle's formula: Ultrasonography: Fetal biometry and estimated date of delivery (EDD) by assessment of BPD, HC, FL \& AC. Expected fetal weight measured by Hadlock formula. Fetal lie and presentation. Exclusion of fetal anomalies. Measurement of both right \& left fetal kidney length. Results: In this study, 255 participants were recruited and assessed for eligibility, 125 women were allocated to perform obstetric ultrasound just 115 completed and were included in the final analysis. The current study found
\end{abstract}


that there was a highly significant positive correlation between gestational age (according to ultrasound parameters) and KL, BDP, HC, AC and FL but Kidney length was the most correlated parameter. Also this study found that there was significant positive correlation between gestational age (according to LMP) and gestational age estimated by BPD, HC, AC, FL and KL with the most significant positive correlation being with KL. Also this study found that there was no significant difference (matched) in KL and Gestational age compared to other parameters. This means that KL is the most accurate parameter for Estimating Gestational age, correlation between MKL and GA by LMP $(\mathrm{r}=0.951, \mathrm{p}=0.000)$ the most significant correlation. In this study, no significant difference was found between gestational age estimated by KL and gestational age in all stages of pregnancy. This means that KL remains accurate as a predictor for gestational age regardless stage of pregnancy. Conclusion: Kidney length can be used as an individual parameter in estimating gestational age.

\section{Keywords}

Kidney Length, Gestational Age, Growth Parameters

\section{Introduction}

An accurate age of fetus plays a pivotal role in obstetric care. Uncertain dates and no assigned ultrasound date in early trimester poses a dilemma in management decision leading to iatrogenic pre or post maturities. In high risk pregnancies like pre-eclampsia, IUGR, GDM, planning termination of pregnancy due to complications or to plan for fetal investigations or therapy requires an accurate gestational age [1].

Even if menstrual history is correct, the exact time of ovulation, fertilization, and implantation cannot be known. Women may undergo several "waves" of follicular development during a normal menstrual cycle, which may mean ovulatory inconsistency during any given cycle. Sperm may survive for 5 to 7 days in the female reproductive tract; a "known" conception date is therefore not completely reliable. Recent studies suggest the ovulation-to-implantation duration can vary by as much as 11 days, and this may affect fetal size and growth [2].

In routine ultrasonography, the ultrasonologist measures the bi-parietal diameter (BPD), head circumference (HC), abdominal circumference (AC) and femur length (FL) in estimating the gestational age (GA) and estimated date of delivery [3]. However as the pregnancy advances these parameters become increasingly unreliable in prediction of GA. Therefore accurate estimation of GA in late second and third trimester still remains problem [4].

Fetal kidney has been shown a steady growth of $1.7 \mathrm{~mm}$ fortnightly (every 2 weeks) throughout pregnancy and is unaffected by growth abnormalities. Various studies have reported that fetal kidney length (FKL) strongly correlates with the gestational age in late trimester. Fetal kidney is easy to identify and measure 
but has not been studied extensively as a biometric index for gestational age estimation, although ultrasound textbooks often have tables of different dimensions [5].

The study aimed to assess the accuracy of the gestational age estimated by mean fetal kidney length compared to multiple growth parameters like BPD, $\mathrm{HC}, \mathrm{AC} \& \mathrm{FL}$ in addition to the actual gestational age derived from the reliable last menstrual period.

\section{Patients and Methods}

\subsection{Study Type}

Diagnostic accuracy hospital based study.

The study was conducted at ultrasound unit; department of Obstetrics and Gynecology, Faculty of Medicine, Ain Shams University from the period of July 2018 to December 2018

A total number of 115 Pregnant women at their third trimester by reliable LMP attended antenatal care clinic at Ain-Shams University Hospital and consented to participate in this study.

Sample size was calculated using PASS ${ }^{\circledR}$ version 11 program, setting the type-1 error $(\alpha)$ at 0.05 and the power (1- $\beta$ ) at 0.9 . Results from a previous study Goyal et al., [5] showed that there was a strong positive correlation between GA and MFKF $(r=0.714)$. Calculation according to these values produced a minimal sample size of 30 cases however, a sample of 115 cases will be recruited to detect a correlation between this two variable with lower correlation coefficient $(r=$ $0.35),(p=0.000)$.

\section{Inclusion Criteria:}

1) All cases with singleton pregnancies in the third trimester (28 to 40 weeks).

2) Women who were sure of the dates of their last menstrual period ( $1^{\text {st }}$ day) and their cycles were regular at least 3 months before pregnancy.

3) Normal antenatal pregnant women with no associated risk factors.

\section{Exclusion Criteria:}

\section{Maternal}

1) Pregnant women with unknown dates.

2) Multiple pregnancies.

3) Patients with high risk pregnancies such as pre-eclampsia, macrosomia, oligohydraminos or polyhydraminos.

4) Gross maternal obesity.

\section{Fetal}

1) Anomalous fetus.

2) IUGR.

3) Off-springs of diabetic mothers.

4) Renal pelvic dilatation of $5 \mathrm{~mm}$ or greater.

5) Renal anomalies and Abnormal renal morphology.

6) Obscured adrenal and renal borders or margins. 


\subsection{Methodology}

The study was conducted at ultrasound special care unit for the fetus; department of Obstetrics and Gynecology, Faculty of Medicine, Ain Shams University from the period of January 2020 to June 2020.

A total number of 115 Pregnant women at their third trimester by reliable LMP attended antenatal care clinic at Ain-Shams University Hospital and consented to participate in this study.

\section{Every woman was subjected to:}

\section{A) History taking:}

- Personal history. name, age, sex, occupation and address.

- Menstrual and obstetric history. Date of LMP, expected date of delivery and gestational age. In addition to history of presence of any menstrual irregularities, duration.

- Past History. of other diseases like hypertension, diabetes mellitus liver or renal diseases, collagen diseases or any other condition that may affect fetal growth.

$>$ Women with regular cycles and excellent dates were determined by asking the patients if they were:

- Sure of their dates.

- Having regular cycles with no history of hormonal contraception administration for the previous 3 cycles.

- Serum pregnancy test confirming pregnancy following amenorrhea.

- First trimester ultrasound confirming pregnancy, gestational age and expected date of delivery.

Expected date of delivery was calculated according to Naegle's formul [12] a:

$$
\mathrm{EDD}=\mathrm{LMP}+7 \text { days }-3 \text { months }+1 \text { year }
$$

Naegele's rule [12] is a standard way of calculating the due date for a pregnancy. The rule estimates the expected date of delivery (EDD) by adding one year, subtracting three months, and adding seven days to the first day of a woman's last menstrual period (LMP). The result is approximately 280 days (40 weeks) from the start of the last menstrual period. Another method is by adding 9 months and 7 days to the first day of the last menstrual period.

Another method is used which is called Parikh's formula is a calculation method that considers cycle duration. Naegele's rule assumes an average cycle length of 28 days, which is not true for everyone. EDD is calculated using Parikh's formula by adding nine months to the start of the last menstrual period, subtracting 21 days, then adding duration of previous cycle.

In our current study, Naegle's formula [12] was used.

\section{B) Medical examination:}

- General:

1) Assessment of vital data (blood pressure, pulse).

2) Estimation of blood sugar level.

- Abdominal examination to assess fundal height. 


\section{C) Ultrasonography:}

1) Fetal biometry and estimated date of delivery (EDD) by assessment of BPD, HC, FL \& AC.

2) Expected fetal weight measured by Hadlock formula.

3) Fetal lie and presentation.

4) Exclusion of fetal anomalies.

5) Measurement of both right \& left fetal kidney length as follows:

The fetus was scanned in transverse plane until the kidney is visualized just below the stomach. The probe is rotated through $90^{\circ}$ to outline the longitudinal axis of the kidney during apnea. The right and left kidney lengths were measured by $2 \mathrm{D}$ ultrasound machine Samsung SonoAceR 5 produced by Samsung Medison CO.LTD.

Each kidney length was measured twice by a single sonographer then the average was taken.

Gestational age was calculated from mean fetal kidney length using normograms by [6].

These values were compared with actual gestational age derived from actual dates taken as a standard.

Statistical methods: Data collected throughout history, basic clinical examination, ultrasound measurements and outcome measures coded, entered and analyzed using Microsoft Excel software. Data were then imported into Statistical Package for the Social Sciences (SPSS version 20.0) (Statistical Package for the Social Sciences) software for analysis. According to the type of data qualitative represent as number and percentage, quantitative continues group represent by mean $\pm S D$, the following tests were used to test differences for significance; difference and association of qualitative variable by Chi square test $\left(\mathrm{X}^{2}\right)$. Differences between parametric paired data by paired $t$ test, correlation by Pearson's correlation and regression by linear regression. $\mathrm{P}$ value was set at $<0.05$ for significant results $\&<0.001$ for high significant result.

\section{Results}

- Assessed for eligibility $(\mathrm{n}=137)$ women with Singleton pregnancies $(28-40$ $\mathrm{w})$, Excluded $(\mathrm{n}=12)$, Not meeting inclusion criteria $(\mathrm{n}=8)(1$ diabetic, 2 hypertensive, was 42 yrs old, 3 twins pregnancy, 1 preterm), Declined to participate $(n=4)$ Allocated to intervention $(n=125)$, Received allocated intervention $(n=115)$, did not receive allocated intervention $(n=10)$, Lost to follow-up (patients refused to underwent US examination) $(n=6)$, Discontinued intervention (patients refused to cooperate) $(n=4)$.

- Age was distributed as $29.14 \pm 4.98$ with minimum 20 and maximum 38 years, GA was distributed as $35.8 \pm 3.26$ with minimum 28 and maximum 41 weeks, majority were multigravida $82.7 \%$, regard abortion $15.6 \%$ had abortion regard delivery $47.8 \%$ had CS and $34.7 \%$ had VD (Table 1 ).

- FL was distributed as $69.83 \pm 6.68$ and BPD was distributed as $88.12 \pm 5.0$ 
and HC \& AC as $310.95 \pm 26.4 \& 312.85 \pm 33.1$ respectively and GA by US was distributed as $35.94 \pm 3.17$ (Table 2).

- Right KL was $35.13 \pm 3.72$ and left was $34.88 \pm 3.72$ and mean KL was $35.19 \pm$ 3.36 (Table 3).

- EFW was distributed as $2743.76 \pm 769.2$ (Table 4).

- All parameters significantly positive correlated with all parameters, with MKL is the most significant parameter along with GA by LMP (Table 5).

- Significant regression with estimated formula equal $Y=a+b X 2517.1$ * $(177.1$ * SC) $($ Table 6).

- There was no significant difference between GA by LMP and estimated GA from FL, BPD, HC, AC or KL, with the MKL most significant. (Table 7).

Table 1. Basic demographic and obstetric data distribution among studied group $(\mathrm{N}=$ 115).

\begin{tabular}{cccc}
\hline \multirow{2}{*}{ Age/years } & Mean \pm SD & \multicolumn{2}{c}{$29.14 \pm 4.98$} \\
& Median (Range) & $28.0(20-38)$ \\
GA/weeks by LMP & Mean \pm SD & \multicolumn{2}{c}{$35.8 \pm 3.26$} \\
& Median (Range) & \multicolumn{2}{c}{$36.1(28-41)$} \\
\hline \multirow{2}{*}{ Gravidity } & PG & N & \% \\
\hline \multirow{2}{*}{ Abortion } & Multigravida & 20 & 17.3 \\
& No & 95 & 82.7 \\
& Yes & 97 & 84.4 \\
Delivery & PG & 18 & 15.6 \\
& CS & 20 & 47.3 \\
& VD & 55 & 34.7 \\
& Total & 40 & 100.0 \\
\hline
\end{tabular}

$\mathrm{PG}=$ primgravida; $\mathrm{CS}=$ cesarean section $; \mathrm{VD}=$ vaginal delivery.

Table 2. Ultrasonography parameters distribution among studied group.

\begin{tabular}{ccc}
\hline FL & Mean \pm SD & $69.83 \pm 6.68$ \\
& Median (Range) & $71.0(56-81)$ \\
BPD & Mean \pm SD & $88.12 \pm 5.0$ \\
& Median (Range) & $89.0(72-96)$ \\
HC & Mean \pm SD & $310.95 \pm 26.4$ \\
& Median (Range) & $319.0(217-352)$ \\
AC & Mean \pm SD & $312.85 \pm 33.1$ \\
& Median (Range) & $312.0(214-370)$ \\
GA by US & Mean \pm SD & $35.98 \pm 3.17$ \\
& Median (Range) & $36.4(28.4-40.4)$ \\
\hline
\end{tabular}

$\mathrm{FL}=$ femur length $\mathrm{BPD}=$ biparital diameter; $\mathrm{HC}=$ head circumference; $\mathrm{AC}=$ abdominal circumference; GA by US = gestational age by ultrasound. 
Table 3. Ultrasonography estimated kidney length distribution among studied group.

\begin{tabular}{cccc}
\hline & RKL & LKL & MKL \\
\hline Mean \pm SD & $35.13 \pm 3.72$ & $34.88 \pm 3.72$ & $35.19 \pm 3.36$ \\
Median (Range) & $35.0(26-43)$ & $35.0(23-42)$ & $35.5(25.5-41.5)$ \\
\hline
\end{tabular}

$\mathrm{RKL}=$ right kidney length; $\mathrm{LKL}=$ left kidney length; $\mathrm{MKL}=$ mean kidney length.

Table 4. Estimated fetal weight distribution among studied group.

\begin{tabular}{cc}
\hline & EFW \\
\hline Mean \pm SD & $2743.76 \pm 769.2$ \\
Median (Range) & $2790.0(1100-4150)$ \\
\hline
\end{tabular}

$\mathrm{EFW}=$ expected fetal weight.

Table 5. Correlations between GA by LMP and other parameter.

\begin{tabular}{|c|c|c|}
\hline & & GA by LMP \\
\hline \multirow{2}{*}{ FL } & $\mathrm{R}$ & $0.884^{\star \star}$ \\
\hline & $\mathrm{P}$ & 0.000 \\
\hline \multirow{2}{*}{ BPD } & $\mathrm{R}$ & $0.753^{\star \star}$ \\
\hline & P & 0.000 \\
\hline \multirow{2}{*}{$\mathrm{HC}$} & $\mathrm{R}$ & $0.810^{* \star}$ \\
\hline & $\mathrm{P}$ & 0.000 \\
\hline \multirow{2}{*}{$\mathrm{AC}$} & $\mathrm{R}$ & $0.828^{* *}$ \\
\hline & $\mathrm{P}$ & 0.000 \\
\hline \multirow{2}{*}{ GA by US } & $\mathrm{R}$ & $0.889^{* *}$ \\
\hline & $\mathrm{P}$ & 0.000 \\
\hline \multirow{2}{*}{ RKL } & $\mathrm{R}$ & $0.932^{* *}$ \\
\hline & $\mathrm{P}$ & 0.000 \\
\hline \multirow{2}{*}{ LKL } & $\mathrm{R}$ & $0.784^{\star *}$ \\
\hline & $\mathrm{P}$ & 0.000 \\
\hline \multirow{2}{*}{ MKL } & $\mathrm{R}$ & $0.951^{\star *}$ \\
\hline & $\mathrm{P}$ & 0.000 \\
\hline \multirow{2}{*}{ EFW } & $\mathrm{R}$ & $0.896^{\star *}$ \\
\hline & $\mathrm{P}$ & 0.000 \\
\hline
\end{tabular}

$\mathrm{FL}=$ femur length $\mathrm{BPD}=$ biparital diameter $\mathrm{HC}=$ head circumference; $\mathrm{AC}=$ abdominal circumference; GA by US = gestational age by ultrasound; $\mathrm{RKL}=$ right kidney length; $\mathrm{LKL}=$ left kidney length; $\mathrm{MKL}=$ mean kidney length; EFW = expected fetal weight; ${ }^{* *}=$ remarkable result.

Table 6. Regression formula for kidney length to predict GA.

\begin{tabular}{cccc}
\hline & $\mathbf{R}^{2}$ & $\mathbf{P}$ & Predict formula \\
\hline FL & 0.92 & $0.00^{\star *}$ & $\left(0.47^{\star} \mathrm{FL}\right)+2.94$ \\
BPD & 0.58 & $0.00^{\star *}$ & $(0.49 * \mathrm{BPD})+-7.4$ \\
HC & 0.65 & $0.00^{\star *}$ & $\left(0.1{ }^{*} \mathrm{HC}\right)+4.77$ \\
\hline
\end{tabular}


Continued

\begin{tabular}{clll}
\hline AC & 0.68 & $0.00^{* *}$ & $\left(0.08^{*} \mathrm{AC}\right)+10.27$ \\
Right kidney length & 0.86 & $0.00^{* *}$ & $\left(0.81{ }^{*} \mathrm{RKL}\right)+7.08$ \\
Left kidney length & 0.67 & $0.00^{* *}$ & $(0.68 * \mathrm{LKL})+11.86$ \\
Mean kidney length & 0.91 & $0.00^{* *}$ & $(0.92 * \mathrm{MKL})+3.38$ \\
\hline
\end{tabular}

$\mathrm{FL}=$ femur length; $\mathrm{BPD}=$ biparital diameter; $\mathrm{HC}=$ head circumference; $\mathrm{AC}=$ abdominal circumference ultrasound; $\mathrm{RKL}=$ right kidney length; $\mathrm{LKL}=$ left kidney length; $\mathrm{MKL}=$ mean kidney length; ${ }^{* *}=$ remarkable result.

Table 7. Paired analysis to estimate difference between actual GA and estimated GA.

\begin{tabular}{cccccc}
\hline & & Mean & Std. Deviation & Paired t & P \\
\hline Pair 1 & GA by LMP & 35.8009 & 3.26240 & -2.560 & $0.008^{*}$ \\
& GA by US & 35.9800 & 3.17310 & & \\
Pair 2 & GA by LMP & 35.8009 & 3.26240 & 0.089 & 0.929 \\
& GA by FL & 35.7607 & 3.14045 & & \\
Pair 3 & GA by LMP & 35.8009 & 3.26240 & 0.053 & 0.958 \\
& GA by BPD & 35.7797 & 2.45013 & & \\
Pair 4 & GA by LMP & 35.8009 & 3.26240 & -0.166 & 0.869 \\
& GA by HC & 35.8657 & 2.64911 & & \\
Pair 5 & GA by LMP & 35.8009 & 3.26240 & 1.283 & 0.202 \\
& GA by AC & 35.2982 & 2.64831 & & \\
Pair 6 & GA by LMP & 35.8009 & 3.26240 & 1.415 & 0.151 \\
& GA by RKL & 35.5427 & 3.01530 & & \\
& GA by LMP & 35.8009 & 3.26240 & 1.470 & 0.144 \\
Pair 7 & GA by LKL & 35.5231 & 2.53437 & & \\
& GA by LMP & 35.8009 & 3.26240 & 0.435 & 0.664 \\
Pair 8 & GA by MKL & 35.7600 & 3.09999 & & \\
\hline
\end{tabular}

$\mathrm{GA}=$ gestational age; $\mathrm{LMP}=$ lastmenstrual period; $\mathrm{US}=$ ultrasound; $\mathrm{FL}=$ femur length; $\mathrm{BPD}=$ biparital diameter; $\mathrm{HC}=$ head circumference; $\mathrm{AC}=$ abdominal circumference; $\mathrm{RKL}=$ right kidney length; $\mathrm{LKL}=$ left kidney length; $\mathrm{MKL}=$ mean kidney length; ${ }^{*}=$ remarkable result.

\section{Discussion}

In this study 255 participants were recruited and assessed for eligibility, 125 women were allocated to perform obstetric ultrasound just 115 completed and were included in the final analysis.

The current study found that there was a highly significant positive correlation between gestational age (according to ultrasound parameters) and KL, BDP, HC, AC and FL but Kidney length was the most correlated parameter.

Also this study found that there was significant positive correlation between gestational age (according to LMP) and gestational age estimated by BPD, HC, AC, FL and KL with the most significant positive correlation being with KL.

Also this study found that there was no significant difference (matched) in KL 
and Gestational age compared to other parameters. This means that KL is the most accurate parameter for Estimating Gestational age.

In this study no significant difference was found between gestational age estimated by KL and gestational age in all stages of pregnancy. This means that KL remains accurate as a predictor for gestational age regardless stage of pregnancy.

\section{Agreed with our study:}

Cohen et al., [6] study was done to measure normal lengths of fetal kidneys sonographically during pregnancy. Gestational ages were determined by last menstrual period and biometry. Strong correlation exists between renal length and gestational age $(r=0.82)$, determined by biparietal diameter, femoral length, and abdominal circumference, and an average of the three. No significant difference was found between right and left renal lengths in fetuses in whom both kidneys were imaged. Average renal lengths are significantly different when compared across the range of gestational ages ( $p$ less than 0.001).

Konje et al. [7] objective was to evaluate the application of kidney length measurement to the determination of gestational age between the 24th and 38th weeks and to compare its accuracy with that of other fetal biometric indices. Seventy-three women with singleton uncomplicated pregnancies underwent standard ultrasound fetal biometry and kidney length measurement every 2 weeks between 24 and 38 weeks' gestation. These measurements were used to date the pregnancies relative to crown-rump length dating between 8 and 10 weeks' gestation. Linear regression models for estimation of gestational age were derived from the biometric indices and kidney length. In addition, stepwise regression models were constructed to determine the best model for determining gestational age between 24 and 38 weeks. Comparisons were then made between the accuracy of these models in the determination of gestational age.

The best model for estimating gestational age in late pregnancy included the variables kidney length, biparietal diameter, head circumference, femur length and abdominal circumference. This model accurately predicted gestational age with a standard error of \pm 8.48 days. A model including kidney length, biparietal diameter, head circumference and femur length accurately predicted gestational age with a standard error of \pm 8.57 days. These models were slightly more accurate than models derived from the biometric indices of biparietal diameter, head circumference and femur length ( \pm 9.87 days), biparietal diameter, head circumference, femur length and abdominal circumference ( \pm 9.45 days) and biparietal diameter and femur length ( \pm 9.9 days). Kidney length and femur length were the most accurate single parameters for predicting gestational age using simple linear regression models $( \pm 10.29$ and 10.96 days, respectively); the abdominal circumference was the least accurate ( \pm 14.54 days) [7].

Toosi and Rezaie-Delu, [8] study aimed to evaluate the normal fetal kidney length (KL) and its correlation with GA. A cross-sectional study on 92 pregnant women between 8th and 10th week of gestation with normal singleton pregnancy underwent standard ultrasound fetal biometry and kidney length measurement. Univariate and multivariate linear regression analysis was used to create a 
predictive equation to estimate GA on the KL and fetobiometry parameters. A significant correlation was found between GA and KL $(r=0.83)$. The best GA predictor was obtained by combining head circumference, fetal biparietal diameter, femur length and KL with a standard error (SE) about 14.2 days. The findings showed that KL measurements combination with other fetal biometric parameters could predict age of pregnancy with a better precision

In Shivalingaiah et al. [1] study, Fetal kidney length has been studied and shown to strongly correlate with the gestational age in late trimesters even in IUGR fetuses $(r=0.85)$. The study evaluated the role of kidney length in determining the gestation age with the study population of 60 pregnant women between 24 - 36 weeks of gestation whose pregnancies were dated accurately by early dating scan. Length of the nearer kidney was measured in centimeters, 4 weekly in the longitudinal axis along with other biometric indices. According to the observations, the mean deviation from the gestational age at all the weeks is least for KL. The result indicates that the kidney length in the present study correlated well with the assigned gestational age and found almost same as all the ultrasound biometric parameters put together.

Bardhan et al., [9] study aimed to determine correlation and regression coefficient of foetal kidney length as a parameter for gestational age and to do its comparative evaluation with other foetal biometric indices. A Prospective observational study was conducted on 200 women aged between 18 to 35 years with normal singleton pregnancies, over a duration of six months from December 2015 to May 2016. Pearson's correlation coefficient value of 0.99 and regression coefficient or slope 1.0 was noted between gestational age and fetal kidney length with a significance $\mathrm{P}<0.001$. The study concluded that Fetal kidney length shows a positive correlation with fetal gestational age, with a linear growth rate throughout pregnancy irrespective of underlying medical condition like intrauterine growth retardation. This study shall prove its worth in cases of engaged fetal head, where it is difficult to measure fetal bi parietal diameter and head circumference as well as in cases of intrauterine growth retardation, macrosomia and malformation where abdominal circumference measurement gives false values.

Chatterjee et al. [10] study aimed to evaluate Foetal kidney length as a parameter for determination of gestational age in pregnancy by ultrasonography. A cross section hospital based study was conducted in the Department of Obstetrics and Gynecology, P.B.M. and Associated Group of Hospitals, attached to Sardar Patel Medical College, Bikaner during study period of one year from 2015 to 2016. 100 pregnant women with known dates of different parity and ages were included in this study. According to the observations, the mean deviation from the gestational age at all the weeks is least for KL. The result indicated that the kidney length in the present study correlated well with the assigned gestational age and found almost same as all the ultrasound biometric parameters put together. The study concluded that Kidney length can be used as an individual parameter in estimating gestational age, especially in later trimesters, where bio- 
metric indices may not be much reliable.

Muhammad et al., [11] found the point of this examination was to assess the criticalness of the fetal kidney length (FKL) for the estimation of gestational age. Method: A cross sectional study was carried out on 399 pregnant women with singleton pregnancies having no complications at Baloch Hospital Lalian. Pregnant women with single fetuses underwent standard ultrasound fetal biometry. The fetal kidney length measurements were performed from 20th weeks to 38th weeks of gestation. These measurements were used to date the pregnencies. Results: A positive linear relationship was found between gestational age (measured by femur length) and length of left kidney. G.A in weeks (measured by femur length $)=25.430+1.912$ (Left Kidney in $\mathrm{cm}$ ) with $\mathrm{R}^{2}=0.939$ and $\mathrm{p}$ value $=0.000$ $<0.05$. Another statistically significant positive linear relationship was found between gestational age (measured by femur length) and length of right kidney. G.A in weeks (measured by femur length) $=13.496+5.727$ (Right Kidney in $\mathrm{cm}$ ) with $\mathrm{R}^{2}=0.939$ and $\mathrm{p}$ value $=0.000<0.05$. Conclusion: $\mathrm{FKL}$ is easy parameter to identify and measure. It is the most accurate single parameter for estimating GA than other biometric indices especially in cases of abnormal fetal growth or certain maternal conditions when the other parameters like BPD, FL, AC and HC are not reliable for assessing GA in the 2nd and 3rd trimester of pregnancy.

\section{Conclusion}

Kidney length can be used as an individual parameter in estimating gestational age.

\section{Conflicts of Interest}

The authors declare no conflicts of interest regarding the publication of this paper.

\section{References}

[1] Shivalingaiah, N., Sowmya, K., Ananya, R., Kanmani, T.R. and Marimuthu, P. (2014) Fetal Kidney Length as a Parameter for Determination of Gestational Age in Pregnancy. International Journal of Reproduction, Contraception, Obstetrics and Gynecology, 3, 424-427. https://doi.org/10.5455/2320-1770.ijrcog20140628

[2] Butt, K. and Lim, K., Diagnostic Imaging Committee (2014) Determination of Gestational Age by Ultrasound. Journal of Obstetrics and Gynaecology Canada, 36, 171-181. https://doi.org/10.1016/S1701-2163(15)30664-2

[3] Gupta, D.P., Gupta, H.P., Zaidi, Saxena, D.K. and Gupta, R.P. (2013) Accuracy in Estimation of Gestational Age in Third Trimester by Fetal Kidney Length in Indian Women. Indian Journal of Clinical Practice, 24, 459-463.

[4] Kaul, I., Vinay, M., Amandeep, K. and Gupta, R. (2012) Role of Fetal Kidney Length in Estimation of Gestational Age. JK Science, 14, 2.

[5] Goyal, L., Agarwal, S., Chandra, S., et al. (2016) Foetal Kidney Length: A Useful Parameter for Ultrasonographic Gestational Age Calculation. National Journal of Integrated Research Medicine, 7, 55-58.

[6] Cohen, H.L., Cooper, J., Eisenberg, P., Mandel, F.S., Gross, B.R., Goldman, M.A., 
Barzel, E. and Rawlinson, K.F. (1991) Normal Length of Fetal Kidneys: Sonographic Study in 397 Obstetric Patients. AJR American Journal of Roentgenology, 157, 545548. https://doi.org/10.2214/ajr.157.3.1872242

[7] Konje, J.C., Abrams, K.R., Bell, S.C. and Taylor, D.J. (2002) Determination of Gestational Age after the 24th Week of Gestation from Fetal Kidney Length Measurements. Ultrasound in Obstetrics \& Gynecology, 19, 592-597.

https://doi.org/10.1046/j.1469-0705.2002.00704.x

[8] Toosi, F.S. and Rezaie-Delu, H. (2013) Evaluation of the Normal Fetal Kidney Length and Its Correlation with Gestational Age. Acta Medica Iranica, 51, 303-306.

[9] Bardhan, J., Ghosh, S.K., Sarkar, K.N. and Sarkar, M. (2016) Fetal Kidney Length as a Parameter for Gestational Age Determination and Its Comparative Evaluation with Other Fetal Biometric Indices. IAIM, 3, 36-44.

[10] Chatterjee, S., Yadav, K., Prakash, P. and Shekhawat, K. (2016) Foetal Kidney Length as a Parameter for Determination of Gestational Age in Pregnancy by Ultrasonography. International Journal of Reproduction, Contraception, Obstetrics and Gynecology, 5, 1949-1952. https://doi.org/10.18203/2320-1770.ijrcog20161696

[11] Akram, M.S., Yousaf, M., Farooqi, U., Arif, N., Riaz, A., Khalid, M., Fatima, M., Gillani, S.A. and Glani, S.M.Y.F. (2019) Estimation of Gestational Age from Fetal Kidney Length in the Second and Third Trimester of Pregnancy by Ultrasonography. Saudi Journal of Medical and Pharmaceutical Sciences, 5, 222-229.

[12] Edwards, K.I. and Itzhak, P. (2020) Estimated Date of Delivery. StatPearls Publishing, StatPearls. 